\title{
Philosophiques
}

\section{Décoloniser le féminisme. Ignorance épistémique, solidarité et réparation}

\section{NAÏMA HAMROUNI}

Volume 44, numéro 1, printemps 2017

URI : https://id.erudit.org/iderudit/1040336ar

DOI : https://doi.org/10.7202/1040336ar

Aller au sommaire du numéro

Éditeur(s)

Société de philosophie du Québec

ISSN

0316-2923 (imprimé)

1492-1391 (numérique)

Découvrir la revue

Citer ce document

Hamrouni, N. (2017). Décoloniser le féminisme. Ignorance épistémique, solidarité et réparation. Philosophiques, 44(1), 145-153.

https://doi.org/10.7202/1040336ar d'utilisation que vous pouvez consulter en ligne.

https://apropos.erudit.org/fr/usagers/politique-dutilisation/ 


\title{
Décoloniser le féminisme. Ignorance épistémique, solidarité et réparation
}

\author{
NAÏMA HAMROUNI \\ Département de science politique \\ Université Laval \\ naima.hamrouni@pol.ulaval.ca
}

Décoloniser le féminisme. Un titre qui fracasse. Qui fait frémir la blanchité. La provoque. Sous-entendu: le féminisme, mouvement social qui émerge d'une juste révolte contre l'oppression, qui se bat pour un monde libre et désenchaîné, ce mouvement, idéaliste, juste et vertueux, il est «colonial » : il colonise, il empiète sur, il écrase. Après leurs terres et leurs corps, les esprits et les idéaux des femmes qui peuplent le monde qui vit toujours dans l'ombre de l'Occident.

Depuis quelques décennies maintenant, il n'est plus admis de parler de LA femme en pensée féministe. LA femme. Comme s'il y avait quelque chose de tel qu'un modèle-type Femme, un standard normatif, une Universelle. Une source unique de l'oppression, une seule stratégie de libération qui vaille. Bien sûr, nous parlons désormais DES femmes. Aussi veut-on marquer par là la diversité des positionnalités, l'intersectionnalité des oppressions, l'hétérogénéité du "groupe Femmes". Passons donc de « $L a$ femme » à "Les femmes »: une transition sémantique exigeante, qui promettait un changement de paradigme ambitieux. Une transition sémantique de façade, qui a en bonne partie englouti «toutes les femmes» dans $\mathrm{La}$ femme, tout en prétendant le contraire, mots à l'appui.

Le féminisme est toujours colonial. "Ledit féminisme, écrit Mestiri, n'a pas vocation à émanciper les femmes, toutes les femmes, mais [...] il cherche à leur imposer un certain mode d'être de manière foncièrement hégémonique et arbitraire, au motif que la liberté doit se concevoir ainsi et pas autrement»(Mestiri, 20I7, p. I03). Cependant, dans Décoloniser le féminisme, Mestiri ne se limite pas à reformuler la critique classiquement adressée au féminisme hégémonique occidental blanc — du type de celles déjà formulées à l'encontre des approches de Susan Moller Okin ou de Martha Nussbaum ${ }^{1}$. La penseuse s'attache aussi, et de manière fine, à souligner le caractère contre-productif des réponses forgées au sein d'un féminisme se voulant "à contre-courant", "non libéral ", "postcolonial» (Mestiri, 20I7, p. I04). Passant au crible le féminisme laïque de la sociologue marocaine Fatima Mernissi qui «oscille entre dénonciation de l'orientalisme

1. Dans le contexte québécois, notre livre, codirigé avec Chantal Maillé, Le sujet du féminisme est-il blanc? Femmes racisées et recherche féministe (2015), participe aussi de ce mouvement critique.

PHILOSOPHIQUES 44/1 — Printemps 2017, p. 145-153 
et tentation iconisante qui peut la faire renouer avec des travers typiquement orientalistes" (chap. II p. 40), le féminisme multiculturaliste de la philosophe américaine d'origine coréenne Sarah Song (Épilogue), jusqu'au féminisme musulman tel qu'il se déploie dans le contexte occidental pour offrir une relecture féministe du Coran (chap. III), la philosophe politique d'origine tunisienne montre que ces féminismes "postcoloniaux", qu'elle qualifie sans détour de "théories-pansement ", n'apportent finalement que des correctifs superficiels à l'oppression subie par les femmes des peuples (toujours) colonisés. Ces variantes du féminisme échouent, malgré leurs honnêtes efforts, à renverser les rapports de domination Nord-Sud Ouest-Est, et à représenter une solution de rechange véritablement émancipatrice pour (toutes) les femmes.

Dans le cadre de cette disputatio, j'aimerais me pencher sur ce que je considère être la proposition au cœur de l'ouvrage de Soumaya Mestiri, soit cette invitation à penser un féminisme transversal, un féminisme de la frontière. Plus précisément, je me pencherai sur le rôle que donne l'auteure à l'éthique du care pour mener à bien le projet de décoloniser le féminisme, de revigorer l'empowerment véritable des femmes des Suds, et de refonder une solidarité entre les femmes du monde qui ne pècherait plus par excès de maternalisme.

$* * *$

Comment le féminisme est-il colonial? Un des cas les plus convaincants traités par Mestiri est celui des stratégies d'empowerment des femmes pauvres des Suds par les ONG les plus puissantes, qui les prennent sous leur aile pour «les émanciper» et, comme le veut l'expression, leur «apprendre à pêcher ». Le verdict de Mestiri est ici sans appel:

Habiliter ou émanciper les femmes pauvres revient en dernière instance à les dépolitiser avec la bénédiction plus ou moins consciente d'une élite féminine elle-même instrumentalisée à cette fin. Loin d'être un "remède au devenirfemme de la pauvreté ", l'approche empowerment donne à voir et incarne tout à la fois la «colonialité du pouvoir $[\ldots] »^{2}$.

Et encore :

Les femmes politiques que l'on forme n'échappent pas à ce schéma muséal: c'est la caricature de la femme leader que l'on cherche ici à promouvoir non pas simplement en la formant, mais en la formatant ${ }^{3}$.

Des phrases qui ont cette superbe de vous lancer un pavé dans la mare de la bonne conscience, il y en a à chaque page de ce livre court mais dense. C'est au chapitre V que la penseuse démonte les approches Gender and

2. Mestiri, 2016, p. I7.

3. Ibid., p. I 8 . 
Development (GAD), qui se présentaient pourtant comme des solutions de loin supérieures aux approches de première génération «Femmes et développement ", mises en vigueur dès le début des années I970, et qui avaient eu le défaut d'évacuer de leurs analyses les rapports sociaux de pouvoir. Ces approches GAD, depuis enseignées partout dans nos Facultés de sciences humaines et sociales comme représentant $L a$ voie vers l'habilitation des femmes les plus pauvres du globe (assimilées à un même groupe homogénéisé, construit comme irresponsable, inculte, mineur, et marchant "d'un même pas vers un telos fédérateur " (p. I I3), ces approches, disais-je, ont été cooptées par les forces idéologiques et politiques néolibérales mises en œuvre par les grandes ONG. Elles cherchent à assimiler les pauvres femmes du monde à la logique du marché conçu comme le levier par excellence de la lutte à la pauvreté. Ces stratégies alimentent non seulement le mythe selon lequel si elles manquent de poisson, ces femmes déshéritées, c'est qu'elles n'ont d'abord jamais appris à pêcher (ce que l'écrivaine franco-algérienne Bouteldja appelait déjà une "analogie faussée ", 20I6), mais elles se trouvent au bout du compte à "produire des citoyens dociles et obéissants, ce qui est $[\ldots]$ à contre-courant des objectifs initiaux de ces [...] expériences d'empowerment» (p. II 7 ).

On a enchaîné les marins au rivage, puis on a coulé leurs chaloupes, puis on a vidé leur mer. Puis, par un tour de force politico-langagier, on est tout de même parvenu à attribuer leur famine à leur inculture, et à nous imposer comme devoir moral d'aller les "habiliter", après avoir campé, dans toute cette triste histoire, le colonisateur dans le rôle du Bon Samaritain. Ce chapitre V, qui rappelle des pages sublimes de Vers une politique de l'amour révolutionnaire de Bouteldja (2016), doit à tout prix être inclus aux sommaires de nos cours d'éthique des relations internationales et de théories de la justice - comme d'autres chapitres d'ailleurs, dont le premier, sur les femmes du fantasmé Kazanistan rawlsien.

Pourtant, le constat de cet échec ne nous invite «ni au pessimisme ni à l'inaction» (p. I I9). Si l'auteure veut en effet exposer les éléments troubles de la notion d'empowerment, qui est marquée par «l'ambivalence fondamentale à vouloir aider l'autre tout en maintenant de manière souvent pernicieuse un rapport de force qui lui est défavorable", c'est pour mieux la réinvestir de son potentiel émancipateur. "Émanciper les femmes de toute forme de tutelle [...] Transformer la société» (p. II9).

Plus d'efforts devront cependant être faits pour nous persuader de cette proposition qui est donc esquissée au chapitre VI suivant: l'éthique du care serait $L e$ vecteur par lequel réhabiliter l'empowerment. Comptant parmi le groupe de philosophes québécoises qui ont cherché à examiner comment le care en tant qu'éthique et politique pourrait être "réinvesti de son plein potentiel critique et féministe» (Hamrouni, 2015) ${ }^{4}$, je suis d'em-

4. Voir Le care: éthique féministe actuelle (2015). 
blée sympathique à cette idée. Cela dit, de mon point de vue, sous sa formulation dominante actuelle, l'éthique du care n'est pas à la hauteur de cette prétention et contient même, en son sein, un fort potentiel d'infériorisation de ceux qui, sous cette approche, sont toujours catégorisés comme les «dépendants» en besoin de «care» que ce care soit donné par leurs proches ou fourni par les institutions publiques qui relèvent de l'État. Mais ma critique ne porte pas sur ce risque inhérent aux éthiques du care articulées autour de la figure du «dépendant» dont je traite ailleurs (2015).

Si le care est vu comme un "outil décolonial », écrit Mestiri, c'est qu'il peut « incarner cette réciprocité que nous recherchions et qui faisait gravement défaut à l'empowerment pour qui il s'agissait ni plus ni moins que de sauver les femmes du Sud»(p. I6). Cet outil, donc, viendrait mettre un terme au maternalisme inhérent aux stratégies d'empowerment qui se retrouvent en pratique à reproduire les rapports de force inégaux entre, d'un côté, occidentales expertes autoproclamées et, d'un autre côté, indigènes néophytes - qui ont du moins été soigneusement dépeintes comme telles. Mais est-ce qu'attirer l'attention sur la nécessaire "réciprocité ", sur le rétablissement d'une égalité entre elles, ne risque pas d'invisibiliser l'essentiel, c'est-à-dire le colonialisme persistant qui nous unit (ou, plus exactement, qui nous sépare) et dont nous tirons chaque jour un avantage disproportionné? Est-ce que la réciprocité ne risque pas d'occulter l'ampleur de la dette que nous avons envers ces femmes?

L'auteure dit vouloir ancrer son propos dans une philosophie sociale plutôt que politique-normative, qui postulerait un idéal à atteindre. Mais alors que l'éthique du care est présentée en dernière instance comme la voie pour penser la réciprocité entre nous toutes et la solidarité bien comprise, nous pouvons être laissées sous l'impression que c'est au déploiement d'une véritable approche normative que nous assistons. En effet, le care, compris comme véritable Théorie critique, nous conduirait bien plutôt vers l'analyse des processus complexes qui font obstacle à l'établissement d'une réciprocité véritable entre femmes du Nord et femmes orientalisées. La théorie du care comprise comme Théorie critique ancrée dans une philosophie sociale nous amènerait —et c'est ce que je défends- à saisir les mécanismes qui maintiennent, à travers les vastes réseaux de l'économie mondialisée, le flux du transfert de care, asymétrique et unilatéral, des peuples subalternes vers les peuples dominants. Ce care invisible dont nous, héritières des entreprises coloniales d'Occident, bénéficions toujours, et qui nous permet de nous maintenir dans cette illusion de l'indépendance, de l'autosuffisance. C'est du moins ce que je soutiens (2012; 20I5).

\section{$* * *$}

La féministe d'Occident, elle la détient, la supériorité morale. Elle est la fille des Lumières, des grandes Révolutions et des Indépendances. Une fille de l'agnosticisme et de la mise à mort de Dieu. De la liberté et de l'égalité, de la 
tolérance et de la démocratie. Une fille des têtes de monarques tranchées. De l'autonomie et du droit de propriété, et de toutes ces formules qui vous promettaient monts et merveilles: "Aie le courage de te servir de ton propre entendement! » Elle est un produit de la Modernité, qui est son berceau et son horizon.

Impériale, cela va de soi. Elle s'exporte, déborde de ses frontières, et emporte dans sa vague - sa première, sa deuxième, sa troisième - les opprimées, les muselées, les excisées, ces Indigènes. Celles qui se couvrent d'obscurité, des pieds à la tête. Et puis celles qui sur l'autre continent mettent au monde des enfants secs, amaigris et mourants. Elle les relèvera, elle les élèvera, elle les sauvera. Ses sœurs et leurs filles.

«L'appel à la sororité, écrivait bell hooks, a [...] souvent été perçu comme une manœuvre manipulatrice et opportuniste des bourgeoises blanches, un vernis rhétorique servant à masquer l'exploitation et l'oppression perpétuées par des femmes sur d'autres femmes ${ }^{5}$.» L'invitation lancée par les blanches à «se joindre " au mouvement féministe mondial dont elles demeureraient évidemment "les propriétaires ${ }^{6}$ ", à adopter la lunette du "genre ", a offert une caution morale à la poursuite par nos gouvernements des politiques impérialistes qui allaient étendre la liberté aux continents en perdition et faire la "guerre à la Terreur ». Nos gouvernements qui seraient alors légitimés de prendre les corps voilés des femmes comme excuses au déferlement militaire sur leurs terres désertiques - et au déchaînement de régiments sur leurs corps. Aussi cet appareillage théorique qu'est le "genre » a-t-il eu l'effet insidieux de braquer ces femmes des «Suds» contre les leurs: leurs pères, leurs frères, leurs maris, noircis, barbarisés, dont tous les crimes sont réécrits comme étant "d'honneur». Le genre, colonial, avait ce pouvoir de les désunir, les hommes et les femmes, pour mieux les miner de l'intérieur, ces colonies brisées.

Depuis que la jeunesse tunisienne s'est enflammée, emportant dans sa vague de la dignité révolutionnaire les grands dictateurs du Maghreb, le débat académique entourant les modalités sous lesquelles une véritable solidarité féministe transnationale pourrait voir le jour a été relancé. C'est un débat auquel le féminisme de la frontière de Mestiri voudrait bien apporter une résolution, du moins, une ouverture. Qu'elle y parvienne est moins sûr. Je vais plus loin en arguant que la solidarité même comprise « de la bonne manière » que l'auteure "appelle de ses vœux» est susceptible de reconduire l'ignorance épistémique de l'histoire coloniale qui sépare les occidentales des femmes arabes, l'ignorance épistémique de la dette que cette histoire a créé pour nous, ses riches héritières. Le vocable de la solidarité doit pour cette

5. Comme l'écrivait la professeure de littérature anglaise et d'études africaines-américaines et militante féministe bell hooks, dans son article "Sororité", 2008, p. I20.

6. hooks, 2008 , p. I25. 
raison être abandonné au profit d'une politique de réparation de l'injustice passée. J'essaierai de m'expliquer dans les lignes qui suivent.

Le féminisme de la frontière, soutient l'auteure, permet de «nous projeter dans la plus parfaite des réciprocités et être ainsi solidaires les unes des autres de la bonne manière ${ }^{7}$ ", c'est-à-dire d'une manière qui en finit avec la présomption des unes et la victimisation des autres, qui reconnaît les différences sans chercher à les effacer ou à les figer, qui prend au sérieux l'intersectionnalité au Nord comme "aux Suds» (trop souvent traités comme un tout homogène). Pour réaliser ce féminisme transversal, il ne s'agit pas, revendique l'auteure, de venir plaquer au centre les savoirs indigènes qui avaient été déclassés, ou de simplement remplacer une épistémologie par une autre, ou encore de reconstruire l'avenir à partir d'une racine nouvelle, purement orientale. Le féminisme transversal, celui qui habite la frontière, place Orient et Occident dans un face à face, à voix et à statut égaux, en même temps qu'il les fait éclater, les "intersectionnalise". Il met en garde aussi bien contre les images angélisées d'un Orient purifié et mystifié que contre la diabolisation excessive d'un Occident dont il faudrait nous protéger des influences de part en part perverses et corruptrices. Ce féminisme transversal, il défait et embrouille les marges et le centre, il déconstruit. Il veut forger une trame narrative nouvelle, certes, mais en glanant ça et là, des éléments des unes et des autres, remises sur un pied d'égalité.

Cette préoccupation, voire cette obsession des femmes privilégiées pour la solidarité transnationale est cependant fascinante. Les femmes sudisées, elles, ne cherchent remarquablement pas à être «solidaires de nous »... Par quelle méprise avons-nous même pu imaginer leur envie de voir briller la «solidarité » dans le regard des épouses de leurs (ex)geôliers? Rappelonsle, la solidarité est ce «rapport existant entre des personnes qui, ayant une communauté d'intérêts, sont liées les unes aux autres". Et encore: le "sentiment d'un devoir moral envers les autres membres d'un groupe, fondé sur l'identité de situation, d'intérêts: Agir par solidarités». Une communauté d'intérêts. La solidarité: ce mot qui invisibilise la dépendance des citoyennes des pays riches vis-à-vis de l'exploitation des citoyennes des pays «misérabilisés » à qui elles doivent tout. Et leur prospérité, et leur puissance militaire, et leur train de vie surconsommé, et leurs programmes sociaux. Solidarité: ce mot qui occulte l'exploitation passée et soutenue de ces terres, de ces corps, de ces forces de travail, de ces sexes, exploitation dont nous bénéficions à chaque jour de nos vies si grasses. Ce mot qui réécrit l'histoire et réunit en une "communauté » (d'intérêts!) la toute-puissance des uns et la déshumanisation des autres. Solidarité: ce mot qui innocente avant de faire appel à un devoir moral surérogatoire: soyez solidaires!

7. Mestiri, 20I7, p. 4. Je souligne.

8. Dictionnaire Le Larousse. En ligne: http://www.larousse.fr/dictionnaires/francais/ solidaritél 733 I 2 ? q=solidarité\# 72484 
Sur l'innocence, Bouteldja (20I6) avait récemment ces mots, limpides:

Vous portez tous ce visage de l'innocence. C'est là votre victoire ultime. Nous sommes coupables, vous êtes innocents. Et vous avez fait de nous les gardiens de votre innocence. [...] Vous faites [...] de l'impunité votre royaume. Vous êtes des anges, parce que vous avez le pouvoir de vous déclarer anges et celui de nous faire barbares (Bouteldja, 2016, p. 30-3I).

Quel est le réquisit de la justice? Reconnaître, d'abord, que notre positionnalité structurellement privilégiée est tirée du récent passé de nos empires colonisateurs et de leur incapacité (entendre "refus ») à réparer le dépouillement qu'ils ont imposé à leurs "colonisés ". Peut-être n'avons-nous pas de «sang sur les mains» au sens littéral du terme, mais nous jouissons quotidiennement d'un statut privilégié sur l'échiquier mondial qui nous permet de tirer avantage de ce que d'autres, dans nos pays, aient (eu) eux-mêmes du sang sur les mains. Boutledja, encore, avait à ce sujet ces mots qui martèlent l'injustice avec précision: "Je n'ai pas de sang sur les mains. Ce serait trop vulgaire. [...]. Mon crime, je le sous-traite» (Bouteldja, 2016: p. 24). Cette image, très forte, nous enjoint à admettre que si nous ne sommes pas coupables au sens juridique du terme, nous ne pouvons nous défausser de notre responsabilité dans le maintien de ces disparités de pouvoir et de richesses structurelles qui ne vont qu'en s'accentuant depuis les Indépendances. Nous bénéficions au quotidien non seulement de cette "sous-traitance» de l'exploitation; nous bénéficions en plus de ce que des mécanismes épistémiques nous amènent à ne plus la voir cette exploitation, nous autorisent à nous en disculper, à nous innocenter. Nous blanchir. C'est tout cela, être Blanc.

Et parfois même, par un revirement sémantique qui tient de l'extraordinaire, ces mécanismes qui brouillent les pistes de la chaîne de sous-traitance nous permettent carrément de nous déclarer Sauveuses. Quel est le réquisit de la justice, demandais-je plus haut? Après avoir admis notre responsabilité quant à la situation dans laquelle se trouvent les femmes des Suds, il s'agit ensuite de reconnaître que le point de départ de nos interactions n'en est pas un d'égalité ou de réciprocité (évidemment), ni même d'iniquités (qui seraient dues au hasard de la naissance, par exemple). Mais que ce point de départ en est un d'asymétrie radicale. Une asymétrie non pas au sens où nous serions, en raison de l'arbitraire de notre naissance en sol prospère, en position avantageuse d'aider, de soutenir, de nous solidariser, mais bien plutôt au sens où nous sommes en dette vis-à-vis des femmes de ces ailleurs barbarisés. Le statut privilégié qui nous pousse irrésistiblement à penser que nous devrions apporter notre aide aux femmes pauvres du monde s'est construit à même leur exploitation.

À partir du moment où le problème d'injustice est ainsi redéfini, la notion de solidarité que mobilise Mestiri, notion qui veut nous replacer dans un face à face "réciproque", doit être mise de côté comme contreproductive, en cela qu'elle nourrit l'ignorance épistémique de la dette qui nous 
incombe $e^{9}$. Avant que la réciprocité que la philosophe appelle de ses vœux ne puisse être rêvée, puis s'installer pour de bon (puisqu'il s'agit bien là d'un idéal normatif qui est présenté au dernier chapitre VI), il faut aussi reconnaître notre responsabilité et repayer la dette que nous avons. Prendre pour point de départ la réciprocité participerait encore de ce refoulement des horreurs de l'histoire et du présent dont nous avons tant profité et qui les ont tant desservies.

Il est évidemment permis de penser que Mestiri et les collègues qui participent à cette disputatio pourraient être d'accord avec moi sur le fond, et répliqueraient alors que la réparation de l'injustice passée représente ce que la solidarité comprise "de la bonne manière ${ }^{10}$ ", comme l'écrit Mestiri, exigerait. Je répondrai ici que les mots ont une importance et que celui de «solidarité » tel qu'il est défini, tel que nous l'entendons communément, et en raison de ce qu'il évoque spontanément dans notre imaginaire moral, nous projette dans une "communauté d'intérêts» avec les femmes tiersmondisées et efface, à travers cette représentation faussée de notre relation à elles, la responsabilité que nous avons en tant qu'occidentales quant aux injustices dont elles font les frais.

La solidarité n'exige en effet aucun renoncement substantiel. L'appel à la solidarité répète cette fable ancienne, selon laquelle c'est en raison d'événements fortuits et malheureux, de catastrophes naturelles, de dictateurs corrompus, ou du patriarcat de leurs mâles, que les femmes arabes sont opprimées. Elle efface le colonialisme. Elle nous empêche de penser le juste remède à l'oppression: la réparation. Cette réparation exigera un renoncement extraordinaire. Et la première étape de ce renoncement sera de laisser tomber le masque de l'Innocence avec lequel nous pouvons encore aujourd'hui défiler au cœur des grandes manifestations, en appui aux Révolutions (que nous aimons imaginer) fleuries contre les dictatures arabes de ce monde. Et penser que notre dette est acquittée.

9. Si l'ignorance est traditionnellement comprise comme un phénomène passif, un simple défaut de connaissance de la part du sujet (par exemple, on ne lui aurait pas appris quelque chose - ici, le colonialisme), ou comme une simple forme de négligence ou un désintérêt (par exemple, on ignorerait le colonialisme, en faisant comme si ça n'était pas là), les travaux en épistémologie de l'ignorance montrent qu'au contraire l'ignorance est dans certains cas mieux comprise comme une pratique épistémique active. Il s'agit d'un désaveu cultivé, nourri, entretenu à travers une culture, une série d'habitudes et de pratiques, qui maintient les inégalités de pouvoir. Au sujet des épistémologies de l'ignorance, voir l'ouvrage collectif phare de Sullivan et Tuana (2007) auquel ont entre autres participé des philosophes de la race comme Charles Mills et Linda Alcoff.

10. Mestiri, 20I7, p. 4. 


\section{Références}

Hooks, bell. "Sororité: la solidarité politique entre les femmes », dans (Elsa Dorlin, dir.) Black Feminism. Anthologie du féminisme africain-américain, 19752000, Paris, L'Harmattan, 2008, p. II3-I34.

Bourgault, Sophie, et Julie Perreault. Le care: éthique féministe actuelle, Montréal, Éditions du Remue-ménage, 2015.

Bouteldja, Houria. Les Blancs, les Juifs et nous. Vers une politique de l'amour révolutionnaire, Paris, La Fabrique, 2016.

Dictionnaire Le Larousse. En ligne: http://www.larousse.fr/dictionnaires/francais/ solidarité/733 I 2 ? q=solidarité\# 72484

Hamrouni, Naïma, et Chantal Maillé (dir.). Le sujet du féminisme est-il blanc? Femmes racisées et recherches féministes, Montréal, Éditions du Remue-ménage, 2015 .

Hamrouni, Naïma. "Vers une théorie politique du care. Entendre le care comme "service rendu" ", dans (S. Bourgault et J. Perreault, dir.), Le care: éthique féministe actuelle, Montréal, Remue-ménage, 20I5, p. 7I-93.

- Le care invisible. Genre, vulnérabilité et domination. Thèse de doctorat. Université catholique de Louvain et Université de Montréal, 20 I 2.

Mestiri, Soumaya. "Précis de Décoloniser le féminisme. Une approche transculturelle», dans Philosophiques, vol. 44, $\mathrm{n}^{\circ} \mathrm{I}, 2017$, p. 103-107.

- Décoloniser le féminisme. Une approche transculturelle, Paris, Vrin, «coll. La vie morale», 2016.

Sullivan, Shannon, et Nancy Tuana. Race and Epistemologies of Ignorance, New York, SUNY Press, 2007. 